\title{
Addendum: Moduli stabilisation and the statistics of SUSY breaking in the landscape
}

\author{
Igor Broeckel, ${ }^{a, b}$ Michele Cicoli, ${ }^{a, b}$ Anshuman Maharana, ${ }^{c}$ Kajal Singh ${ }^{c}$ \\ and Kuver Sinha ${ }^{d}$ \\ ${ }^{a}$ Dipartimento di Fisica e Astronomia, Universitá di Bologna, \\ via Irnerio 46, 40126 Bologna, Italy \\ ${ }^{b}$ INFN - Sezione di Bologna, \\ viale Berti Pichat 6/2, 40127 Bologna, Italy \\ ${ }^{c}$ Harish-Chandra Research Institute, HBNI, \\ Jhunsi, Allahabad, UP 211019, India \\ ${ }^{d}$ Department of Physics and Astronomy, University of Oklahoma, \\ Norman, OK 73019, U.S.A. \\ E-mail: igor.broeckel@bo.infn.it, michele.cicoli@unibo.it, \\ anshumanmaharana@hri.res.in, kajalsingh@hri.res.in, \\ kuver.sinha@ou.edu
}

AdDENDUM TO: JHEP10(2020)015

ArXiv EPrint: 2007.04327

\section{More acknowledgments}

AM is supported in part by the SERB, DST, Government of India by the grant MTR/2019/000267.

Open Access. This article is distributed under the terms of the Creative Commons Attribution License (CC-BY 4.0), which permits any use, distribution and reproduction in any medium, provided the original author(s) and source are credited. 